\section{A new tube for use in the fluorescein string test: Localization of oesophageal and gastric bleeding sites with or without the use of radiography}

FRED E. PITTMAN From the Gastroenterology Division, Department of Medicine, Medical University of South Carolina, Charleston, South Carolina, and the Medical Section, Veterans Administration Hospital, Charleston, South Carolina, USA

It is generally accepted that early accurate localization of the site of haemorrhage is a most desirable goal in the management of patients bleeding from the gastrointestinal tract. The fluorescein string test, described originally by Traphagen and Karlan (1958), has received wide acceptance as a safe and accurate method for localizing the site of bleeding from the upper gastrointestinal tract (Haynes and Pittman, 1960; Haynes, Pittman, and Christakis, 1960; Glaser and Charles, 1963; Pittman, 1964; Reisberg and Haubric, 1965; Nissenbaum, Attia, DiBianco, and Groisser, 1965). To perform this test, a radiopaque marked tape or tube is passed orally into the upper intestine and a radiograph of the upper abdomen is obtained to ascertain the position of the device. Twenty $\mathrm{ml}$ of a $5 \%$ solution of sodium fluorescein is then given intravenously $(3.5 \mathrm{ml}$ per $\mathrm{kg}$ body weight for infants and children). After four minutes, the device is withdrawn and examined for fluorescence with an ultraviolet lamp equipped with a Wood's filter. The position of the fluorescence and the upper level of blood are noted and the anatomical position of the bleeding site is ascertained by matching these areas with the radiopaque markers on this radiograph (Haynes, Pittman, and Christakis, 1960).

In early experience with the fluorescein string test, it was found that many patients had great difficulty swallowing the flexible tube. For this reason, a semirigid, radioopaque marked polyethylene tube covered by a sheath of white, non-fluorescent cotton material was designed for the test (Pittman, 1965). It was found that this tube could be passed easily and rapidly into the stomach, even when the patient's cooperation was minimal.

Received for publication 12 April 1973.
The semi-rigid tube has also been used to perform 'limited' fluorescein string tests (Pittman, 1965). The 'limited' test is helpful in determining rapidly if bleeding is occurring from the lower oesophagus, diagnostic information which should be available as soon as possible if the patient has suspected or proven oesophageal varices. In such patients, it is highly desirable to avoid the use of an oesophageal balloon tamponade in the absence of proven bleeding from the lower oesophagus (Bennett, Baker, and Baker, 1952; Conn, 1958; Merigan, Hollister, Gryska, Starkey, and Davidson, 1960).

Because patients are sometimes too ill to transport to the radiology department, or because it is difficult to obtain a radiograph of the upper abdomen for other reasons, a device was designed which permits the performance of the 'limited' fluorescein string test with or without the use of $x$-ray localization. This device (see fig) consists of a polyethylene tube covered by a sheath of white, non-fluorescent cotton

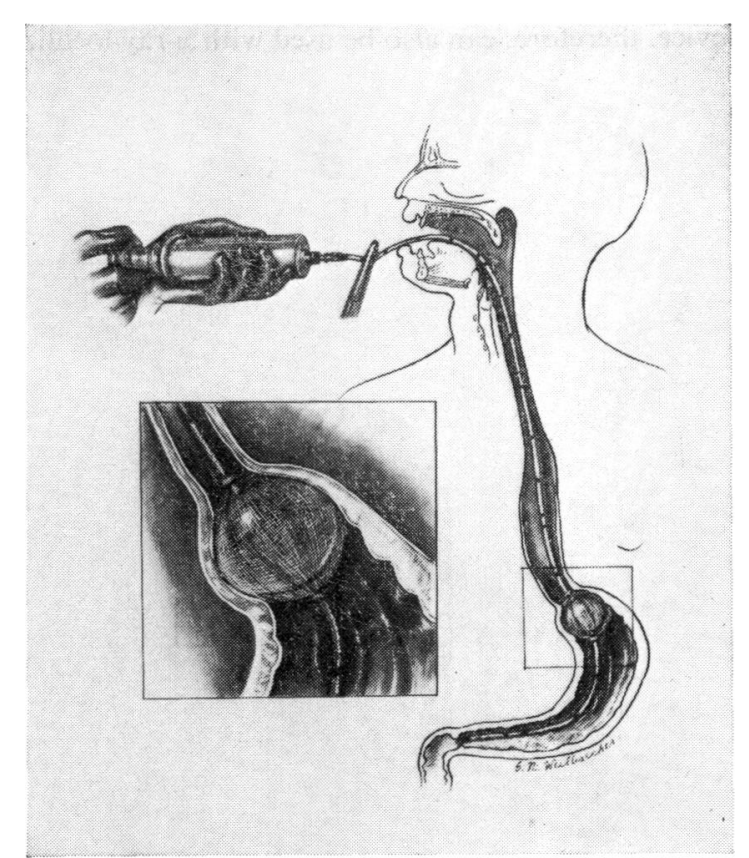

Fig Drawing of the gastrointestinal string type IV: oesophago-gastric bleeding tube which shows the balloon inflated in the gastric fundus. The tube has been clamped after introduction of air into the balloon. 
material. A soft rubber balloon is attached $25 \mathrm{~cm}$ above the distal end of this tube. This balloon is covered with webbed cotton material which provides an absorbent surface and prevents excessive inflation of the balloon. The tube is passed orally into the stomach. One hundred $\mathrm{ml}$ of air is then introduced into the balloon with a syringe attached to the Luer-lock fitting at the proximal end of the device. Egress of the air is prevented by clamping the tube. The tube is then withdrawn until the balloon meets with resistance at the cardio-oesophageal junction. The tube may be held in this position by taping it to the side of the patient's face. Sodium fluorescein is injected intravenously. After four minutes the air is removed by aspirating with the syringe and the tube is withdrawn. The tube is then examined in the usual way (Pittman, 1964). Fluorescence found on the tube above the balloon or at the upper surface of the balloon indicates the likelihood of bleeding from oesophageal varices or other lesions at the cardio-oesophageal junction. The absence of fluorescence in the patient bleeding actively at the time of the test rules out oesophageal or gastric varices as the source of such bleeding and indicates that oesophageal tamponade should not be attempted.

Although this device was designed to be used without radiology, it is marked longitudinally and horizontally with radioopaque monofilament. The device, therefore, can also be used with $x$-ray localiz- ation to confirm the exact position before injection of the fluorescein. Since even minimal movement of the device is prevented by the balloon, the accuracy of localization of the bleeding site is increased.

\section{References}

Bennett, H. D., Baker, L., and Baker, L. A. (1952). Complications in the use of esophageal compression balloons (Sengstaken tube). Arch. intern. Med., 90, 196-200.

Conn, H. O. (1958). Hazards attending the use of esophageal tamponade. New Engl. J. Med., 259, 701-707.

Glaser, G. L., and Charles, R. (1963). The fluorescein string test in upper gastrointestinal tract bleeding. Amer. J. dig., Dis., 8, 292-298.

Haynes, W. F., and Pittman, F. E. (1960). Application of the fluorescein string test in 32 cases of upper gastrointestinal hemorrhage. Gastroenterology, 38, 690-697.

Haynes, W. F., Jr., Pittman, F. E., and Christakis, G. (1960). Location of site of upper gastrointestinal tract hemorrhage by the fluorescein string test. Surgery, 48, 821-827.

Merigan, T. C., Hollister, R. M., Gryska, P. F., Starkey, G. W. B., and Davidson, C. S. (1960). Gastrointestinal bleeding with cirrhosis: a study of 172 episodes in 158 patients. New Engl. J. Med., 263, 579-585.

Nissenbaum, G., Attia, A., DiBianco, J., and Groisser, V. W. (1965). A new device (diagnostotube) for the localization of upper gastrointestinal bleeding. Gastroenterology, 49, 662-666.

Pittman, F. E. (1964). The fluorescein string test: an analysis of its use and relationship to barium studies of the upper gastrointestinal tract in 122 cases of gastrointestinal tract hemorrhage. Ann. intern. Med., 60, 418-429.

Pittman, F. E. (1965). A rapid-entry tube for use in the fluorescein string test. Lancet, 1, 1308-1309.

Reisberg, I. R., and Haubric, W. S. (1965). Localizing obscure upper gastrointestinal bleeding by the fluorescein string test. Henry Ford Hosp. med Bull., 13, 265-270.

Traphagen, D. W., and Karlan, M. (1958). Fluorescein string test for localization of upper gastrointestinal hemorrhage: a preliminary report. Surgery, 44, 644-645. 